\title{
Intramolecular Asymmetric Heck Reactions: Evidence for Dynamic Kinetic Resolution Effects
}

\author{
Michael C. McDermott, ${ }^{\dagger}$ G. Richard Stephenson, ${ }^{*, \dagger}$ \\ David L. Hughes, ${ }^{\dagger}$ Andrew J. Walkington ${ }^{\ddagger}$ \\ ${ }^{\dagger}$ Wolfson Materials and Catalysis Centre, School of Chemical Sciences and Pharmacy, \\ University of East Anglia, Norwich, Norfolk NR4 7TJ, UK \\ ${ }^{\ddagger}$ GlaxoSmithKline, Medicines Research Centre, Gunnels Wood Road, Stevenage, \\ Hertfordshire SG1 2NY, UK \\ g.r.stephenson@uea.ac.uk
}

\section{Supporting Information}

General Procedure for the Measurement of Conversion ${ }^{1}$ from 1 into 2:

For conversion measurements, the samples prepared for the chiral HPLC were analysed at $220 \mathrm{~nm}$ using an Agilent 1100 HPLC system equipped with a $50 \mathrm{~mm}$ x $2 \mathrm{~mm}$ Phenomenex Luna $3 \mu \mathrm{C} 18(2)$ column, in which the mobile phase was a gradient from water to acetonitrile/water (19:1).

General Procedure for the Determination of Enantiomeric Excess ${ }^{1}$ of 2 by Chiral HPLC: The Agilent 1100 series system used for the chiral analysis consisted of a pump, degasser unit, column heater set to $40{ }^{\circ} \mathrm{C}$ and a diode array detector that was set to $254 \mathrm{~nm}$. This HPLC system was equipped with a Chiracel OJ chiral column $(4.6 \mathrm{~mm}$ x $250 \mathrm{~mm})$ supplied by Chirex, and prepared with the eluent system of hexane/ethanol (99.5:0.5). The HPLC system was programmed for the specific chiral HPLC analysis of $\mathbf{2}$, over a run time of 60 minutes, at a flow rate of $1 \mathrm{~mL} \mathrm{~min}^{-1}$ and for the number of samples to be analysed. The samples were prepared in THF $(0.75 \mathrm{~mL})$ and transferred to the chiral HPLC system using a Gilson 233 syringe pump programmed using ReactArray software supplied by Anachem. The chiral HPLC trace data was obtained and recorded by Atlas analytical software during the course of the reaction overnight, and was available once all the chiral HPLC traces had been obtained. The ees generated from these chiral HPLC traces were compared to those generated using chiral shift experiments with the same sample of $\mathbf{2}$, and calibrated accordingly.

\footnotetext{
General Procedure for the Determination of Enantiomeric Excess ${ }^{2,3}$ by Chiral Shift Experiments:

An NMR sample was prepared using Heck product $\mathbf{2}$ or $\mathbf{4}$ (approximately $15 \mathrm{mg}$ ) and deuterated chloroform $(0.75 \mathrm{~mL})$, then the initial ${ }^{1} \mathrm{H}$ NMR spectrum was obtained. To this NMR sample was added a few drops of a solution of the chiral shift reagent, tris [3-heptafluoropropylhydroxymethylene)(+)-camphorato]europium(III), in deuterated chloroform (1M), and the ${ }^{1} \mathrm{H}$ NMR spectrum was again obtained. The addition of the chiral shift reagent solution was repeated until a suitable separation of the peaks representing the two enantiomers in the ${ }^{1} \mathrm{H}$ NMR spectrum was obtained. The ees were calculated using the magnitude of the integration of the two peaks.
}

(1) McDermott, M. C. Ph.D. Thesis, University of East Anglia, 2005.

(2) Sellarajah, S. Ph.D. Thesis, University of East Anglia, 2001.

(3) Ashimori, A.; Matsuura, T.; Overman, L. E.; Poon, D. J. J. Am. Chem. Soc. 1998, 120, 6477-6487. 


\section{Synthesis of bis-2-methylbut-2-enoic acid (2-iodophenyl)imide (3):}
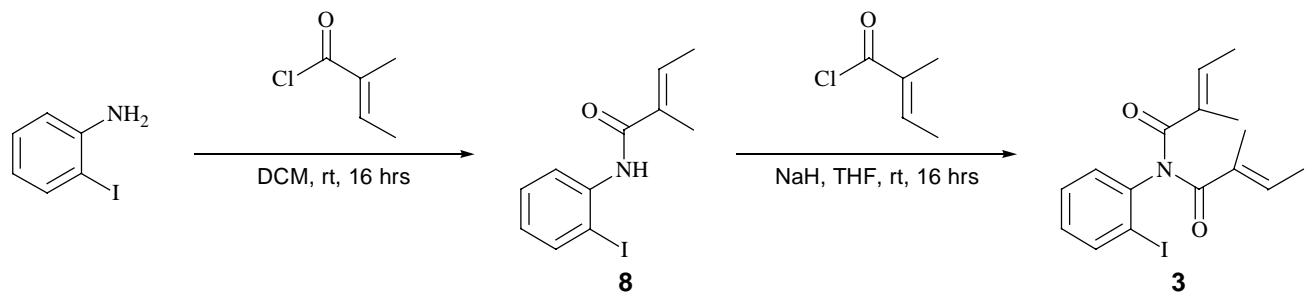

An oven-dried round bottomed flask was charged with 2-iodoaniline (15 g, $68.49 \mathrm{mmol})$ and dry DCM $(40 \mathrm{~mL})$. The aniline solution was stirred and cooled to $0{ }^{\circ} \mathrm{C}$ before a solution of 2-methylbut-2-enoyl chloride $^{4}(8.93 \mathrm{~g}, 75.34 \mathrm{mmol})$ in DCM $(20 \mathrm{~mL})$ was added dropwise via a syringe. The solution was allowed to warm to room temperature and stirred for 16 hours. The reaction mixture was washed with aqueous hydrochloric acid $(2 \mathrm{M}, 2 \times 50 \mathrm{~mL})$. Aqueous sodium hydroxide solution $(2 \mathrm{M}, 50 \mathrm{~mL})$ was added to the organic layer, which was then stirred for 30 minutes at room temperature. The organic layer was separated and the aqueous layer was extracted with DCM $(2 \times 50 \mathrm{~mL})$. The organic extracts were combined, dried over magnesium sulfate, filtered and the solvent removed in vacuo. Purification via column chromatography on silica using hexane/ethyl acetate $(4: 1)$ as the eluent and then recrystallisation from hexane/diethyl ether (20:1) gave 2-methylbut-2-enoic acid (2-iodophenyl)amide ${ }^{5}$ (8) as colourless needles $(14.57 \mathrm{~g}, 71 \%)$; MP 46-48 ${ }^{\circ} \mathrm{C} ; \delta_{\mathrm{H}}\left(400 \mathrm{MHz}, \mathrm{CDCl}_{3}\right) 8.26(1 \mathrm{H}, \mathrm{d}, J$ 8.4, aromatic), $7.79(1 \mathrm{H}$, br s, $\mathrm{NH}), 7.70(1 \mathrm{H}, \mathrm{d}, J 8.0$, aromatic $), 7.26(1 \mathrm{H}, \mathrm{dd}, J 8.2,7.4$, aromatic), 6.76 $\left(1 \mathrm{H}, \mathrm{dd}, J\right.$ 8.0, 7.3, aromatic), $6.63\left(1 \mathrm{H}, \mathrm{q}, J\right.$ 6.9, $\left.\mathrm{CH}_{3} \mathrm{CH}=\mathrm{CCH}_{3} \mathrm{R}\right), 1.94(3 \mathrm{H}, \mathrm{d}, J$ 1.0, $\left.\mathrm{CH}_{3} \mathrm{CH}=\mathrm{CCH}_{3} \mathrm{R}\right), 1.78\left(3 \mathrm{H}, \mathrm{d}, J\right.$ 7.0, $\left.\mathrm{CH}_{3} \mathrm{CH}=\mathrm{CCH}_{3} \mathrm{R}\right) ; \delta_{\mathrm{C}}\left(75 \mathrm{MHz}, \mathrm{CDCl}_{3}\right) 167.2(C=\mathrm{O}), 138.8$, 138.6, 132.8, 132.4, 129.4, 125.7, 121.7, $90.0(\mathrm{C}-\mathrm{I}), 14.2\left(\mathrm{CH}_{3}\right), 12.4\left(\mathrm{CH}_{3}\right)$.

A $50 \mathrm{~mL}$ round-bottomed flask was charged with 2-methylbut-2-enoic acid (2-iodophenyl)amide (8) (5 $\mathrm{g}, 16.60 \mathrm{mmol})$ in THF (20 mL). Sodium hydride (60\% in oil, $797 \mathrm{mg}, 19.93 \mathrm{mmol})$ was carefully added to the solution, which was then stirred for 15-30 minutes at room temperature. 2-Methylbut-2enoyl chloride ${ }^{4}(2.95 \mathrm{~g}, 24.91 \mathrm{mmol})$ in THF $(10 \mathrm{~mL})$ was added to the suspension dropwise via a syringe. After stirring for 16 hours at room temperature the reaction mixture was transferred to a 250 $\mathrm{mL}$ round-bottomed flask, diethyl ether $(50 \mathrm{~mL})$ was added and the reaction was quenched with distilled water $(50 \mathrm{~mL})$ over 30 minutes. The organic layer was separated, washed with distilled water $(50 \mathrm{~mL})$, dried over magnesium sulfate, filtered and the solvent removed in vacuo. Purification via column chromatography on silica using hexane/ethyl acetate (6:1) as the eluent, filtration under reduced pressure using hexane as the eluent and then recrystallisation from hexane/ethyl acetate (10:1) gave bis-2-methylbut-2-enoic acid (2-iodophenyl)imide (3) as a colourless solid (1.49 g, $23 \%$ ); MP $80-81{ }^{\circ} \mathrm{C} ; v_{\max }($ solid $) / \mathrm{cm}^{-1} 2917,2856,1695,1657,1640,1467,1438,1428,1382,1349,1329,1260$, $1241,1138,1085,1021,976,942,866,753,764,733,708,649,626 ; \delta_{\mathrm{H}}\left(300 \mathrm{MHz}, \mathrm{CDCl}_{3}\right) 7.83(1 \mathrm{H}$, $\mathrm{d}, J$ 8.1, aromatic), $7.29\left(1 \mathrm{H}, \mathrm{t}, J 7.5\right.$, aromatic), 6.98-6.93 $\left(2 \mathrm{H}, \mathrm{dq}, J 7.0,1.4,2 \times \mathrm{CH}_{3} \mathrm{CH} \mathrm{CCH}_{3} \mathrm{R}\right)$, $1.74\left(6 \mathrm{H}, \mathrm{s}, 2 \times \mathrm{CH}_{3} \mathrm{CH}=\mathrm{CCH} H_{3} \mathrm{R}\right), 1.44\left(6 \mathrm{H}, \mathrm{d}, J 7.0,2 \times \mathrm{CH}_{3} \mathrm{CH}=\mathrm{CCH}_{3} \mathrm{R}\right) ; \delta_{\mathrm{C}}\left(75 \mathrm{MHz}, \mathrm{CDCl}_{3}\right) 174.7$ $(C=\mathrm{O}), 142.6,140.4,135.6,134.3,129.5,129.4,98.9(\mathrm{C}-\mathrm{I}), 13.9\left(\mathrm{CCH}_{3}\right), 13.1\left(\mathrm{CCH}_{3}\right) ; \mathrm{m} / z(\mathrm{CI}) 466$ $\left(\mathrm{M}+\mathrm{C}_{5} \mathrm{H}_{7} \mathrm{O}, 4 \%\right), 401\left(\mathrm{M}+\mathrm{NH}_{4}^{+}, 88\right), 384\left(\mathrm{M}+\mathrm{H}^{+}, 100\right), 355$ (4), 340 (2), 319 (11), 302 (7), 286 (26), 275 (27), 258 (18), 242 (16), 193 (4), 176 (3), 160 (15), 117 (7), 102 (2), 83 (13); m/z (EI) 383 (M, 3\%), 355 (22), 340 (14), 327 (11), 284 (43), 256 (M-I, 4), 245 (2), 230 (5), 228 (21), 174 ( $\left.\mathrm{C}_{5} \mathrm{H}_{7} \mathrm{OI}, 13\right), 83$ $\left(\mathrm{C}_{5} \mathrm{H}_{7} \mathrm{O}, 100\right), 76(4), 55\left(\mathrm{C}_{4} \mathrm{H}_{7}, 97\right)$; HRMS: Found: $384.0450 \mathrm{C}_{16} \mathrm{H}_{19} \mathrm{O}_{2} \mathrm{NI}\left(\mathrm{M}+\mathrm{H}^{+}\right)$Requires 384.0455.

(4) Haase, K.; Hoffman, H. M. R. Synthesis, 1981, 715-719; Che, Q.; Snider, B. B. Org. Lett., 2004, 6, 2877-2880.

(5) Clark, A. J.; Jones, K. Tetrahedron, 1992, 48, 675-6882. 


\section{Synthesis of $(R A C)$-3-methyl-3-vinyl-1,3-dihydroindol-2-one-(2'-methylbut-2'-enoic acid)imide (4):}
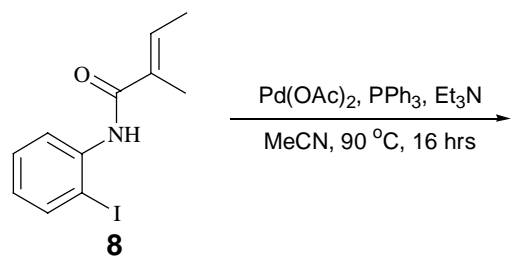
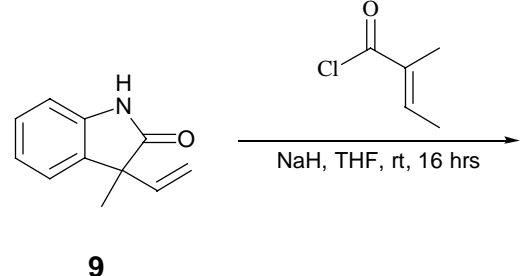

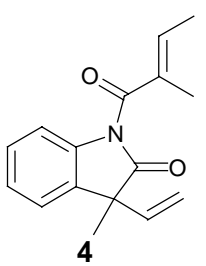

An oven-dried Schlenk tube was charged with palladium(II) acetate $(28 \mathrm{mg}, 0.125 \mathrm{mmol})$, triphenylphosphine $(65 \mathrm{mg}, 0.25 \mathrm{mmol})$ and triethylamine $(0.7 \mathrm{~mL}, 4.98 \mathrm{mmol})$, the reaction vessel flushed with nitrogen and the reagents dissolved in dry acetonitrile $(12 \mathrm{~mL})$. The reaction mixture was placed in an oil bath preheated to $50{ }^{\circ} \mathrm{C}$ and stirred, whilst gradually increasing the temperature. After the reaction reaches $65{ }^{\circ} \mathrm{C}$ (after approximately 15 minutes), or when the solution turns dark red in colour, 2-methylbut-2-enoic acid (2-iodophenyl) $\operatorname{amide}^{5}(\mathbf{8})(750 \mathrm{mg}, 2.49 \mathrm{mmol})$ was added. The reaction mixture was heated to reflux $\left(90{ }^{\circ} \mathrm{C}\right)$ and stirred for 16 hours. After cooling to room temperature, hexane $(50 \mathrm{~mL})$ was added to the mixture. The solution was then washed through celite using diethyl ether as the solvent. The resulting filtrate was dried over magnesium sulfate, filtered and the solvent removed in vacuo. Purification via column chromatography on silica using DCM as the eluent and then recrystallisation from hexane gave 3-methyl-3-vinyl-1,3-dihydroindol-2-one (9) as a colourless solid (180 mg, $42 \%$ ); MP 96- $97{ }^{\circ} \mathrm{C} ; v_{\max }$ (thin film) $/ \mathrm{cm}^{-1} 3215$ (br), 3090, 2973, 2927, 2871, 1711, 1618, 1601, 1472, 1485, 1450, 1408, 1369, 1329, 1295, 1267, 1242, 1199, 1106, 1016, 993, 924 , $757,746,704,648 ; \delta_{\mathrm{H}}\left(300 \mathrm{MHz}, \mathrm{CDCl}_{3}\right) 8.82(1 \mathrm{H}, \mathrm{br} \mathrm{s}, \mathrm{NH}), 7.19-7.09(2 \mathrm{H}, \mathrm{m}$, aromatics $), 7.02(1 \mathrm{H}$, ddd, $J 7.7,7.5,1.0$, aromatic), $6.90\left(1 \mathrm{H}, \mathrm{d}, J 7.7\right.$, aromatic), $5.90\left(1 \mathrm{H}, \mathrm{dd}, J 17.1,10.5, \mathrm{CCH}=\mathrm{CH}_{2}\right)$, $5.13(1 \mathrm{H}, \mathrm{dd}, J 10.4,0.7, \mathrm{CCH}=\mathrm{CHH}), 5.11(1 \mathrm{H}, \mathrm{dd}, J 17.2,0.8, \mathrm{CCH}=\mathrm{CH} H), 1.46\left(3 \mathrm{H}, \mathrm{s}, \mathrm{CCH}_{3}\right) ; \delta_{\mathrm{C}}$ (75 MHz, $\left.\mathrm{CDCl}_{3}\right)$ 181.6 (C=O), 140.3, 137.9, 133.3, 128.1, 124.2, 122.6, 115.6, 110.2, $51.7\left(\mathrm{CCH}_{2}\right)$, $22.2\left(\mathrm{CCH}_{2}\right) ; \mathrm{m} / z(\mathrm{CI}) 192(12 \%), 191\left(\mathrm{M}+\mathrm{NH}_{4}{ }^{+}, 100\right), 176(11), 174\left(\mathrm{M}+\mathrm{H}^{+}, 38\right), 165(3), 160(6)$, 158 (3), 148 (2), 144 (3), 130 (2); m/z (EI) 173 (M, 74\%), 158 (22), 144 (77), $130\left(\mathrm{M}_{-} \mathrm{C}_{2} \mathrm{H}_{3} \mathrm{O}, 100\right)$, 128 (21), 117 (18), 115 (26), 103 (18), 91 (14), 89 (20), 77 (29); HRMS: Found: 191.1179. $\mathrm{C}_{11} \mathrm{H}_{15} \mathrm{ON}_{2}$ $\left(\mathrm{M}+\mathrm{NH}_{4}{ }^{+}\right)$Requires 191.1179.

A $25 \mathrm{~mL}$ round-bottomed flask was charged with 3-methyl-3-vinyl-1,3-dihydroindol-2-one (9) (400 $\mathrm{mg}, 2.31 \mathrm{mmol})$ in THF ( $8 \mathrm{~mL}$ ). Sodium hydride (60\% in oil, $139 \mathrm{mg}, 3.46 \mathrm{mmol})$ was carefully added to the solution, which was then stirred for 10 minutes at room temperature. 2-Methylbut-2-enoyl chloride $^{4}(411 \mathrm{mg}, 3.46 \mathrm{mmol})$ in THF $(2 \mathrm{~mL})$ was added to the suspension dropwise via a syringe. In order to remove any remaining acid chloride a sample of the crude product $(100 \mathrm{mg})$ was dissolved in diethyl ether $(20 \mathrm{~mL})$ and stirred with aqueous sodium hydroxide solution $(0.5 \mathrm{M}, 20 \mathrm{~mL})$. The solution was extracted with diethyl ether $(20 \mathrm{~mL} \mathrm{x} 2)$, then the organic layer was dried over magnesium sulfate, filtered and the solvent removed in vacuo. Purification via column chromatography on silica using hexane/ethyl acetate (10:1) as the eluent gave 3-methyl-3-vinyl-1,3-dihydroindol-2-one-(2'methylbut-2'-enoic acid)imide (4) as a clear oil (36 mg, 6\%); $v_{\max }$ (thin film) $/ \mathrm{cm}^{-1} 3087,3053,3011$, 2972, 2927, 2856, 1755, 1693, 1650, 1606, 1480, 1465, 1451, 1380, 1345, 1301, 1276, 1174, 1127, $1105,1055,1024,928,861,834,795,762,725,682,648,532,489,474,466,448,428,419,410 ; \delta_{\mathrm{H}}$ $\left(300 \mathrm{MHz}, \mathrm{CDCl}_{3}\right) 7.64(1 \mathrm{H}, \mathrm{m}$, aromatic), 7.29-7.21 $(1 \mathrm{H}, \mathrm{m}$, aromatic), 7.16-7.10 $(2 \mathrm{H}, \mathrm{m}$, aromatics), $6.31\left(1 \mathrm{H}, \mathrm{q}, J 7.0, \mathrm{C}=\mathrm{CHCH}_{3}\right), 5.90\left(1 \mathrm{H}, \mathrm{dd}, J 17.2,10.3, \mathrm{CCH}=\mathrm{CH}_{2}\right), 5.15(1 \mathrm{H}, \mathrm{d}, J 10.3$, $\mathrm{CCH}=\mathrm{CHH}), 5.03(1 \mathrm{H}, \mathrm{d}, J 17.3, \mathrm{CCH}=\mathrm{CH} H), 1.85\left(3 \mathrm{H}, \mathrm{d}, J 1.1, \mathrm{C}=\mathrm{CHCH}_{3}\right) 1.77(3 \mathrm{H}, \mathrm{dd}, J$ 7.0, 1.1, $\left.\mathrm{C}\left(\mathrm{CH}_{3}\right)=\mathrm{CHCH}_{3}\right), 1.48\left(3 \mathrm{H}, \mathrm{s}, \mathrm{CCH}_{3}\right) ; \delta_{\mathrm{C}}\left(75 \mathrm{MHz}, \mathrm{CDCl}_{3}\right) 178.1(\mathrm{C}=\mathrm{O}), 171.4(\mathrm{C}=\mathrm{O}), 139.6,138.0$, 137.2, 133.3, 132.0, 128.4, 124.6, 124.0, 116.3, 114.7, $29.6\left(\mathrm{CH}_{3}\right), 22.9\left(\mathrm{CH}_{3}\right), 14.3\left(\mathrm{CH}_{3}\right), 12.7\left(\mathrm{CH}_{3}\right)$; $\mathrm{m} / \mathrm{z}$ (CI) $273\left(\mathrm{M}+\mathrm{NH}_{4}{ }^{+}, 100 \%\right), 256\left(\mathrm{M}+\mathrm{H}^{+}, 85\right), 227$ (5), $212(2), 200$ (2), 193 (7), 191 (25), 176 (12), $174\left(\mathrm{M}-\mathrm{C}_{5} \mathrm{H}_{5} \mathrm{O}, 24\right), 160$ (14), 158 (12), 144 (11), 132 (4), 119 (13), 117 (18), 102 (17), 100 (12), 85 (42), 83 (20); m/z (EI) 255 (M, 3\%), 227 (5), 212 (4), 173 (3), 154 (3), 143 (8), 128 (8), 115 (13), 97 (7), $89(8), 83\left(\mathrm{C}_{5} \mathrm{H}_{7} \mathrm{O}, 100\right), 77(12), 55\left(\mathrm{C}_{4} \mathrm{H}_{7}, 100\right)$; HRMS: Found: $256.1327 . \mathrm{C}_{16} \mathrm{H}_{18} \mathrm{NO}_{2}$ Requires 256.1332 . 


\section{Synthesis of enantiomerically enhanced 3-methyl-3-vinyl-1,3-dihydroindol-2-one-(2'- methylbut-2'-enoic acid)imide (4):}<smiles>CC=C1C/C(=C/C)C(=O)N(c2ccccc2I)C1=O</smiles>

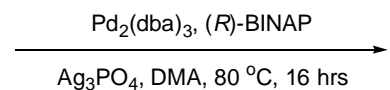

An oven-dried Schlenk tube was charged with tris(dibenzylideneacetone)dipalladium(0) (12 mg, 13 $\mu \mathrm{mol}),(R)$-BINAP $(16 \mathrm{mg}, 26 \mu \mathrm{mol})$ and silver phosphate $(218 \mathrm{mg}, 0.52 \mathrm{mmol})$, the reaction vessel flushed with nitrogen and the reagents dissolved in dry DMA $(1 \mathrm{~mL})$. The reaction mixture was placed in an oil bath preheated to $50{ }^{\circ} \mathrm{C}$ and stirred, whilst gradually increasing the temperature. After the reaction reaches $80{ }^{\circ} \mathrm{C}$, bis-2-methylbut-2-enoic acid (2-iodophenyl)imide (3) (100 mg, $0.26 \mathrm{mmol}$ ) was added. The reaction mixture was heated at $80{ }^{\circ} \mathrm{C}$ and stirred for 16 hours. After cooling to room temperature, ethyl acetate $(5 \mathrm{~mL})$ was added to the mixture. The reaction mixture was filtered through silica and celite using ethyl acetate as the eluent. The resulting solution was washed with aqueous hydrochloric acid $(2 \mathrm{M}, 50 \mathrm{~mL})$ and brine $(50 \mathrm{~mL})$, dried over magnesium sulfate, filtered and the solvent removed in vacuo. Purification via column chromatography on silica using hexane/diethyl ether (4:1) as the eluent gave 3-methyl-3-vinyl-1,3-dihydroindol-2-one-(2'-methylbut-2'-enoic acid)imide ( $100 \%$ conversion), the spectra of which were consistent with the previously acquired data for 4 . The ees were obtained from chiral shift NMR experiments.

\section{Variable Temperature ${ }^{1} \mathrm{H}$ NMR Spectra for $1:^{1}$}

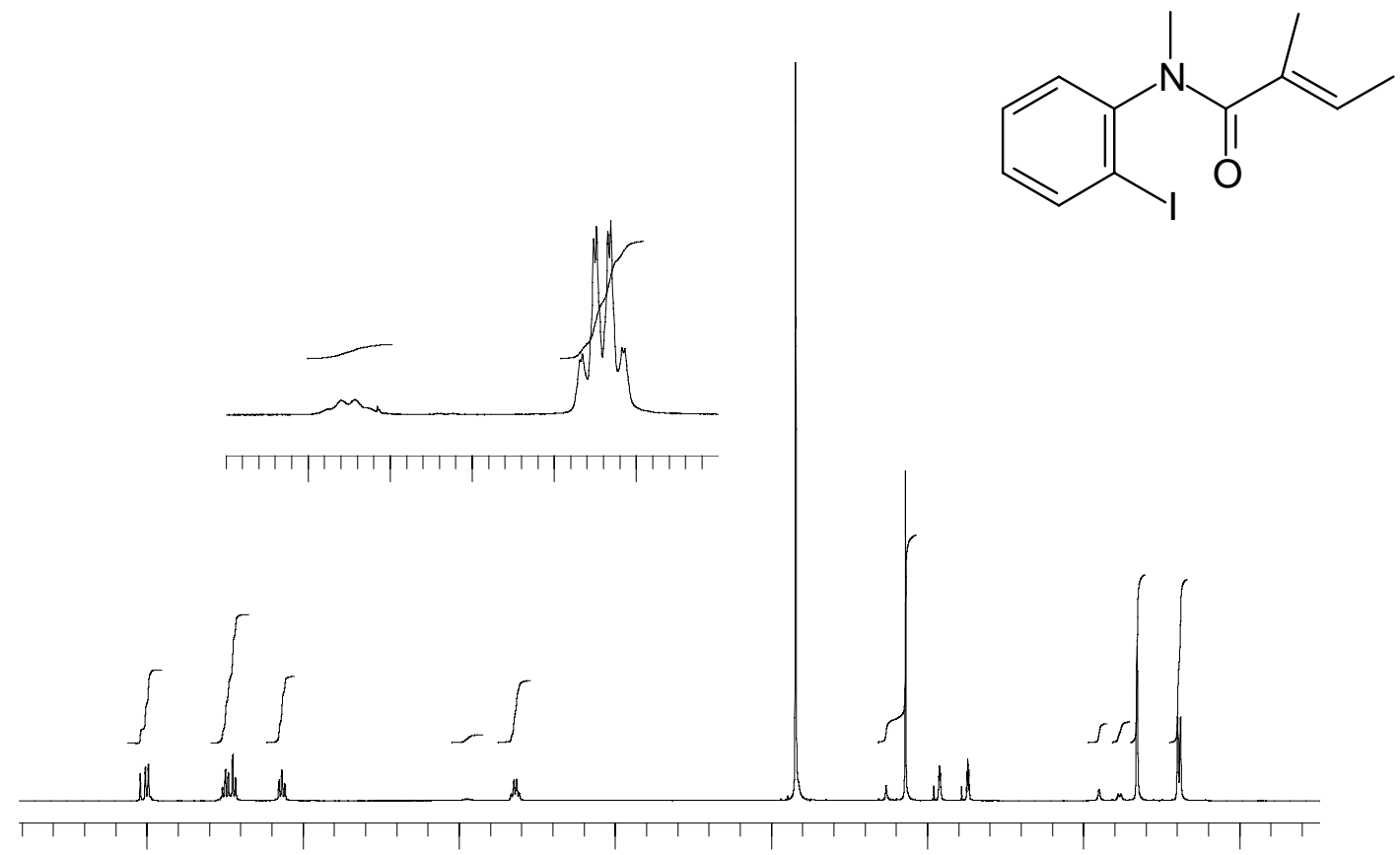

Figure 1: Full NMR spectrum obtained at $-20{ }^{\circ} \mathrm{C}$, using DMF-d $\mathrm{d}_{7}$ as the solvent. 


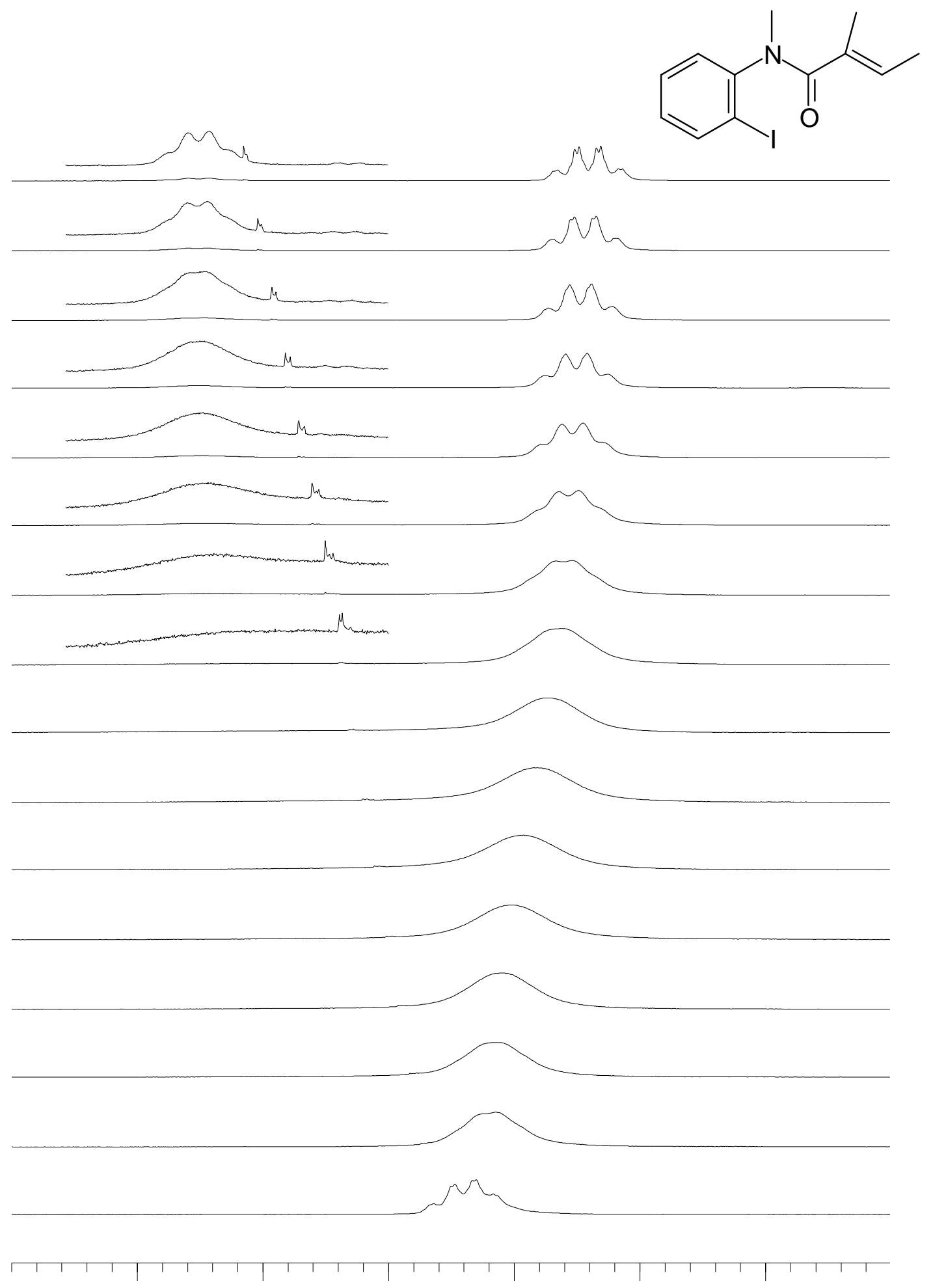

Figure 2: Expansions of VT NMR spectra obtained between $-20{ }^{\circ} \mathrm{C}$ and $80{ }^{\circ} \mathrm{C}$, using DMF- $\mathrm{d}_{7}$ as the solvent (inserts are magnified x 13 on the vertical scale in the region $\delta=6.1-5.8 \mathrm{ppm}$ ).

- S5 - 


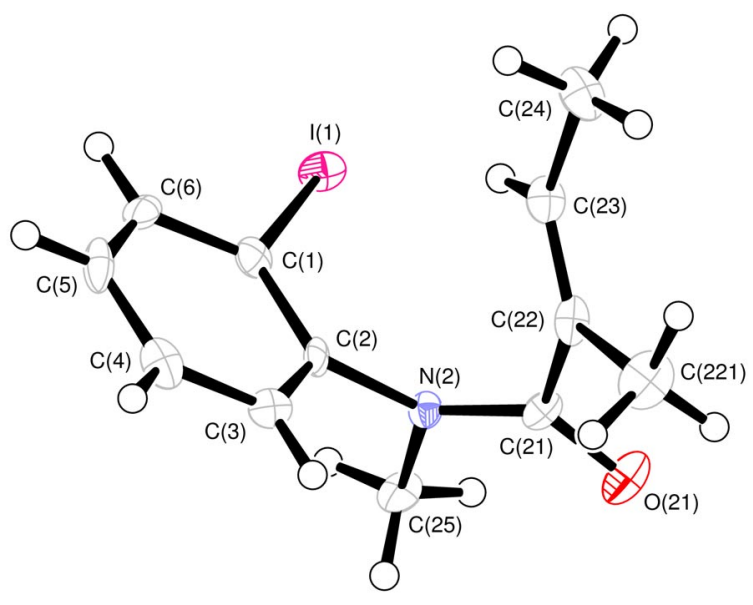

ORTEP drawing of $(P)$-1 from Figure 1

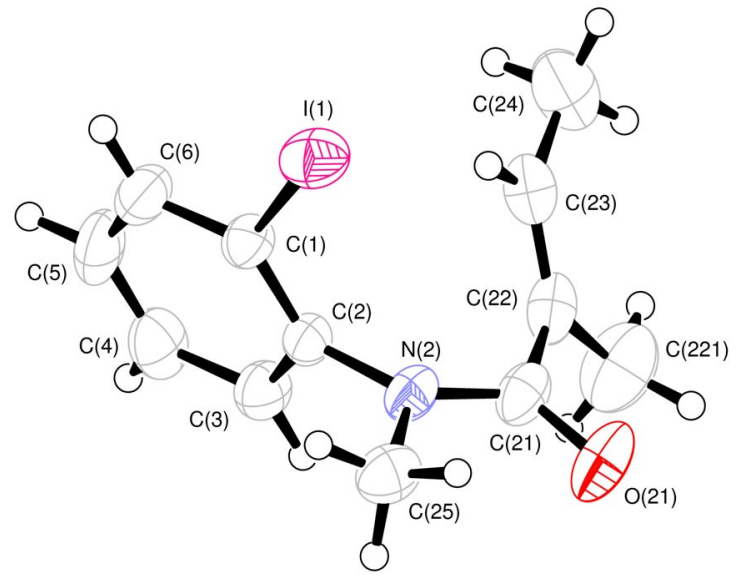

ORTEP drawing of $(M)$-1 from Figure 1 

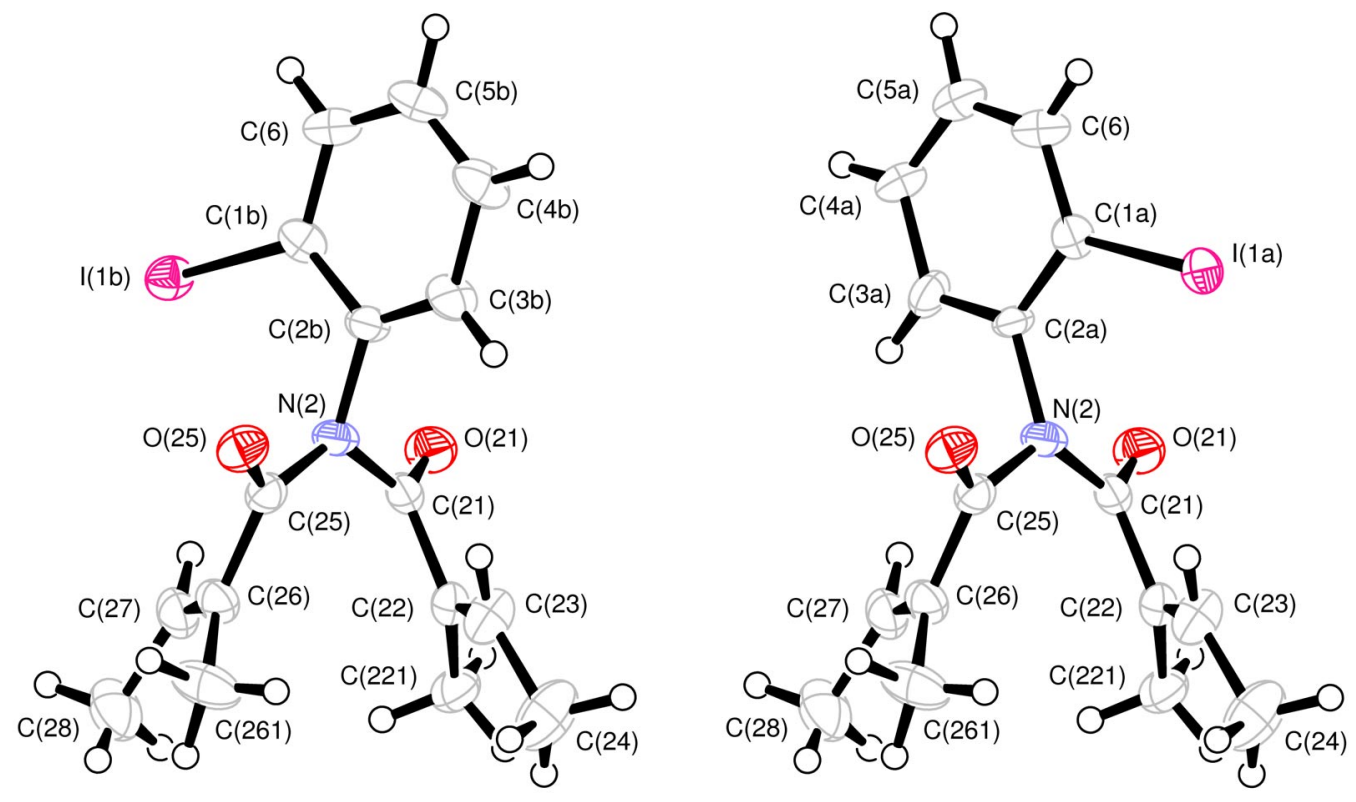

ORTEP drawings of $\mathbf{3}$ from Figure 2 